\title{
Evidence-Based Practices, Ethical Considerations, and Advocacy Efforts to Reduce Mental Health Stigma in Veterans with Post-Traumatic Stress Disorder
}

\section{Adam J McTighe*}

Department of Psychology, Georgia State University, USA

*Corresponding author: Adam J McTighe, Department of Psychology, Georgia State University, USA, Tel: +1 404-413-2000; E-mail: adampsyd@gmail.com Rec date: September 07, 2017; Acc date: October 24, 2017; Pub date: October 26, 2017

Copyright: (C) 2017 McTighe AJ. This is an open-access article distributed under the terms of the creative commons attribution license, which permits unrestricted use, distribution, and reproduction in any medium, provided the original author and source are credited.

\section{Abstract}

Military personnel who see combat are exposed to traumatic experiences beyond what most civilians ever see. These events pose significant and imminent danger of physical injury and death; those lucky enough to survive combat risk developing mental and physical health issues upon their return. Problems common to veterans include depression, substance abuse, and heart disease, but the most pervasive is post-traumatic stress disorder (PTSD). This paper will examine the internal and external factors that influence the development of PTSD, discuss current evidence-based practices, and address the stigma of mental health care. A discussion of the ethical questions that face many clinicians treating veteran populations follows. Finally, this paper will address life-long learning opportunities and suggest advocacy strategies to reduce the stigma associated with mental health care.

Keywords: PTSD; Veteran; Stigma; Evidenced-based treatment; Mental health

\section{Literature Review}

\section{Mental health stigma in veterans with post-traumatic stress disorder}

When soldiers enter combat zones, they are exposed to the constant risk of death. They not only witness bombings, shootings, and other violence, but are asked to commit such violent acts themselves. Such events would be traumatic for anyone; despite their training, soldiers are human beings, and they too experience trauma. Many develop debilitating mental health complications. This paper addresses one of the most prevalent of these, post-traumatic stress disorder (PTSD) [1-3].

PTSD differs from many other mental illnesses in that it is directly related to the way that a specific traumatic experience is processed. PTSD is manifested in negative physiological, emotional, and behavioral changes related directly to the experience except exhibited some time later [4]. Trauma is generalized to stimuli through secondorder classical conditioning and negatively reinforced by avoidance [5]. Once this occurs, complications result, such as heightened arousal states, inability to complete tasks, inability to withstand environmental cues, or reliving the trauma [6].

Many veterans experience PTSD as a personal defect or deficit that they feel they should be able to overcome [7]. This is not the case. Veterans deserve the chance to gain awareness and understanding that PTSD is not a character flaw, but rather a serious, real, and treatable mental health condition. Veterans do not form their perceptions in a vacuum. They are responding to widespread misunderstanding and stigma associated with mental health care [8].

This is evident in reports that only $23-40 \%$ of veterans who carry a mental health diagnosis seek treatment [9]. By comprehensively addressing the stigma of mental health care and better identification of salient risk factors among the veteran population, the behavioral health field can gain accurate prevalence rates, ameliorate suffering through advancing best practices, and address needs through advocacy.

\section{Prevalence rates}

Veterans of the two largest ongoing US military operations, Operation Iraqi Freedom (OIF) and Operation Enduring Freedom (OEF), have shown high PSTD rates, among other complications [10]. Research indicates, remarkably, that one out of three returning combat veterans will experience some form of health or mental health issue, such as major depression, PTSD, aggressive ideation, interpersonal conflict, and suicidal ideation [11]. These numbers are not unique to current engagements; previous wars have also produced significant health concerns.

Magruder and Yeager [12] found (as per the National Vietnam Veterans' Readjustment Study, NVVRS) an estimated PTSD prevalence of roughly $9-19 \%$ for combat veterans deployed in Vietnam, compared to rates of $1-13 \%$ among control groups of non-deployed individuals. The authors found similar results when comparing PTSD rates in more recent OIF/OEF-deployed combat veterans, ranging from 5-20\%, while rates in non-deployed control groups ranged from 3-9\%. Adjusted two-year prevalence rates increased four to seven times following the Iraq invasion. These rates are significant when compared to the general population, which has an estimated PTSD rate of $2.5 \%$, with a one-year rate ranging from $0.5-2.1 \%$ as indicated by the DSMIV-TR.

Seal et al. [2] found that of the nearly 300,000 OIE/OEF veterans using VA services, roughly $37 \%$ received a mental health diagnosis, almost 22\% of which were diagnoses of PTSD. Overall, Magruder and Yeager [12] found that, regardless of war era, the risk of PTSD development increases $1.5-3.5 \%$ when soldiers engage in combat. 


\section{Physical and mental health risks}

War veterans are $2-3 \%$ more likely to develop significant physical and mental health complications than civilian populations [1]. From physical rehabilitation due to bodily injury to cognitive rehabilitation due to traumatic brain injury (TBI), the physical and mental rehabilitation after combat injuries can be exhausting. Injured veterans need to work on physical strength while simultaneously adapting mentally to their new functional abilities. This can be an arduous psychological process.

Schnurr, Lunney, and Sengupta [13] found that PTSD is a significant indicator of worse overall physical health. Veterans also have a significantly higher prevalence of cardiovascular disease due to higher rates of risky behaviors such as smoking, substance use and abuse [10], and poor overall health care [2]. These behaviors are reinforced through maladaptive coping. Other results of poor coping seen in veteran populations include an inability to control or express anger [14], depression [15], and suicide [16]. Psychoeducation and treatment are the first steps in reducing complications. Until then, internal and external factors should be monitored so soldiers can potentially avoid such risks.

\section{Endogenous and exogenous factors}

The simple fact of participation in war may itself indicate that an individual is at risk for developing a psychological disorder [17]. However, the most influential factor is trauma history, which increases the risk for both physical disorders (i.e., heart disease) and psychological disorders (i.e., anxiety, depression, substance abuse, and PTSD) [13]. Other factors are addressed below.

Endogenous factors: From an endogenous perspective, three variables come into play: age, gender, and culture [18]. Veterans younger than 25-years-old have a significantly greater chance of developing PTSD as compared to those older than 40-years-old [2]. Women are more likely to develop arousal or depressive affects, whereas men are more likely to develop substance abuse and avoidance issues [10]. Research indicates close to five percent of women in the military experience rape or sexual assault [19]. Many of these assaults are never reported and survivors are at high risk for developing PTSD.

Sexual preference has also been identified as a risk. Dutra et al. [20] found that lesbian, gay, bisexual or transgendered (LGBT) soldiers have developed PTSD and other health issues (such as depression, anxiety) from harassment from fellow soldiers due to their sexual orientation. Data on convictions do not exist because hate crimes on military bases often go unreported [21]. It is crucial that these issues be addressed in research so clinicians can target presenting problems more accurately and develop appropriate treatment planning.

In many cultures, receiving a mental health diagnosis or seeking therapy are considered signs of weakness or moral defect. Latinos are more likely to amplify symptoms and endorse cluster A traits (i. e. psychosis, paranoia), whereas African Americans tend to be more attenuated [22]. Both perceive treatment as unnecessary [7]. Such perceptions make it difficult for veterans from these cultures to seek or continue treatment. Young Latino men and women have been identified as the most at risk military personal of developing physical (i.e., heart disease) and mental (i.e., PTSD) health complications.

Perhaps the most notable endogenous factor has only just been recently discovered: biomarkers. Van Zuiden et al. [17] measured, in a prospective study of OEF vets, glucocorticoid receptors (GRs) in peripheral blood cells. GRs regulate and control immune response, metabolism, and development. One of the main hormones involved in the process is cortisol, which is released as a result of stress. The authors found that per 1,000 GRs there was a 7.5\% increase in PTSD development. This study suggests a pre-susceptibility for health complications as well as highlighting a need for future research on the subject.

Exogenous factors: Of the exogenous factors, social support is the most important. There is no such thing as too much social support, whether it comes from family, a spouse, or the community. Too little support has been shown to significantly increase the risk of developing and maintaining healthy functioning [23]. However, Jakupcak et al. [16] found that PTSD may reduce the positive effects of social support and allow for suicidal ideation and/or other self-injurious behaviors.

There are factors that have been shown to significantly increase the risk of suicide and self-destructive behaviors; of these, the most significant is social punishment. This includes military demotions, punitive discharges, and drug-related discharges [24]. This is a serious concern, particularly since a dishonorable discharge prohibits the veteran from accessing VA benefits.

Another external risk, and one fundamental to the present discussion, is combat exposure [10]. Maguen et al. [14] found that killing, either directly or indirectly, is a significant predictor of substance use and abuse, anger control problems, relationship issues, and PTSD. The authors also report that combat survivors risk TBIs, which have been linked to dementia-making combat one of the most detrimental exogenous health risks.

The most significant exogenous risk is the stigma of mental health. Stigma creates a negative connotation and infers a personal flaw and inability that goes against social or cultural norms [9]. Research suggests that some veterans do not seek treatment due to concerns about social consequences and discomfort around seeking help [8], and being labeled as weak [7]. Stigma then becomes both an endogenous and exogenous factor that increases the chances of problem development and maintenance.

\section{Current Research}

Research indicates that veteran populations show an increase in visits to primary care physicians, use of medication, and psychotherapies [18] thus masking symptomatology and comorbidity. As a result, researchers have agreed that there is a need to better define mental health burdens as they relate to prevalence, severity, and utilization of health options as a main concern in the veteran population [12]. Improving our understanding of these factors can help us to arrive at a consensus on best practices for treatment-and normalization of treatment is a crucial step toward reducing the stigma veterans feel when approaching the mental health system.

This section discusses four approaches to treatment. They are coping skills, behavioral treatments, cognitive therapies, and eye movement desensitization and reprocessing (EMDR) [25]. It concludes with a novel method of delivering treatment to veterans.

Effective treatment protocols include a multi-dimensional approach involving psychopharmacology, psychotherapy, and psychoeducation [3]. Psychopharmacology alone does not yield the most successful results; research indicates that cognitive, behavioral, and cognitivebehavioral therapy (CBT) can be efficacious by themselves $[4,15,26]$, and treatment can be done remotely. 
Coping. Coping creates the ability to appropriately manage, adapt, and accept situations and is learned through psychoeducation [27]. Clinicians help clients label and identify triggers and behavioral patterns that negatively affect their functioning and mood states. Once achieved, clients gain awareness and are better able to manage problematic situations.

One other proven method to build adaptive coping comes from general education [23]. Bryant [23] suggests that educational benefits that allow returning soldiers the ability to go college, finish their GEDs, or attend trade schools yield significant changes in health and/or functioning. Education allows for mental health to be acknowledged appropriately and handled in a classroom environment-a key factor in reducing stigma.

\section{Behavior}

Prolonged exposure (PE) and response prevention (RP) therapies address avoidance behaviors through a systematic process [28]. By development of systematic units of discomfort (SUDs), feared and avoided stimuli are ranked, and then engaged until the fear response is extinguished. This can either be done in real-world situations or through guided imagery; however, combat zones cannot be recreated, requiring use of guided imagery. Relaxation and breathing retraining are incorporated as a part of the $\mathrm{PE} / \mathrm{RP}$ package and help reinforce new learning [29]. PE/RP maintain treatment gains by instilling hope, attaining mastery over fear by discriminating between safe and unsafe environments, improving daily functioning, and providing a better quality of life [30].

\section{Cognitive}

PTSD prevents individuals from functioning by causing them to develop negative automatic thoughts and inaccurate schemas. In order to treat the client effectively, the faulty core beliefs must be identified. Cognitive restructuring achieves this by replacing the cognitive distortions with realistic, accurate beliefs. Clients become aware of his or her faulty thinking, challenging them client through positive selftalk, and then replacing the negative statements with positive solutions [5].

Cognitive Processing Therapy (CPT) is a variation of CBT. It is manualized and has been shown to be efficacious as well as brief, requiring only twelve structured individual or group sessions [27]. Psychoeducation plays a crucial role in teaching the client to better understand the power behind his or her thoughts and feelings. The goal of the intervention is to help the client modify the meaning of the traumatic event and decrease avoidance associated with the experience. An example of CPT is illustrated below in a case conceptualization.

\section{EMDR}

EMDR has been shown to be efficacious, but is at the center of controversy [25]. EMDR bilaterally stimulates the brain, through eye movement and ear stimulation, in an attempt to reprocess the trauma and appropriately categorize it by labeling new memories. It is without a sound theoretical basis, lacks standardized treatment procedures, and has had mixed results in both military and rape traumas [31].

Karlin et al. [6] indicate that due to the VA's push for specialized treatment for veterans, both CPT and PE have been validated and supported as the evidence-based practices of choice. Regardless of treatment, accurate, appropriate, and clinically sound case conceptualization is the most important factor in determining programming [28].

\section{Image re-scripting}

Image re-scripting has been a long-established practice in cognitive psychotherapy, however, it has more recently been identified as a valuable intervention for PTSD [32]. Treatment involves exploration of potentially triggering images that then become modified across different environments, to ultimately alter the fundamental heuristic beneath the trauma. Clinicians' work with those affect to decrease distress tolerance and process through past negative experiences by acknowledging the images left from them, in order to accept and move beyond the past in a healthy direction [33].

\section{HBT}

Recent research suggests that home-based telehealth (HBT) services have been shown to be as efficacious as traditional psychotherapies [30]. HBT is an approach that can be combined with options outlined above, because it is a form of delivery rather than a type of therapy. HBT allows professionals to build rapport, structure sessions, and realize treatment gains without meeting face-to-face [30,26]. An example of HBT is the Elko Telehealth Outreach Clinic. It was created to allow rural veterans greater access to all levels of VA services through video conferencing [34]. It demonstrates significant advancement in serving veterans with basic health and mental health services (such as psychotherapy and medication checks) that would otherwise be unavailable to them because of their geographic location.

\section{Discussion}

\section{Ethical considerations to be followed}

Combat veterans constitute a population of significant size that has a great need for clinical treatment. Because of the stigma surrounding mental health treatment, it may be difficult for clinicians to connect with them. Many utilize advertising, but they must contend with ethical questions surrounding how to market their services. Veterans are vulnerable to underhanded marketing promises of a healthy and quick readjustment to civilian life. Two of the major ethical principles and codes of conduct that should be addressed when clinicians market their services are accuracy and objectivity, and never engage in face-toface solicitation [35].

\section{Accuracy and objectivity}

Clinicians must appropriately acknowledge their professional level of training and competency in order to market their services to veterans. Public statements must be objective and honest depictions of treatment possibilities and must avoid intentional misunderstandings such as "get well quick." Accurate and objective public statements are a crucial step in correcting the stigma developed by the mass media [36] as well as pharmaceutical corporations [37]. Negative imagery of people suffering from mental problems promulgate stigma and decrease the chances of seeking help. These stereotypes are an unfortunate side effect of unethical profit-driven advances towards vulnerable individuals which is why the psychological community must emphasize accurate and objective advertisements. 


\section{Direct solicitation}

It is troublesome enough that negative stereotypes and pharmaceutical commercials satiate mass media, but it is a whole other issue when direct solicitation takes place. Clinicians must conceal their identity and never intentionally offer services to a veteran. Instead, indirect methods of advertising are recommended (such as brochures or newspaper ads). This will ensure that practices are available as offered and that veterans initiate the treatment they receive. In doing so, clinicians help to build an image of mental health practitioners as trustworthy and principled, thus eroding the stigma surrounding their profession.

\section{Lifelong learning}

Clinicians who treat veterans need continuing education (CE) to learn how to best address their unique behavioral health needs [6]. This is achieved through reading relevant journals, attending seminars, and registering with support organizations.

Journals provide new research and promote best-practices. They also reinforce the local-scientist-practitioner model, a valuable tool when working in geographically and culturally specific veteran populations. The Journal of Trauma and Treatment, The Journal of Traumatic Stress, Psychological Trauma: Theory, Research, Practice, and Policy, and The Journal of Anxiety Disorders are valuable resources. Clinicians can register for CE courses, local seminars, and workshops through the American Psychological Association (APA.org) and local VA hospitals-an important step in staying current with contemporary techniques.

For those clinicians who have not received military training, it is crucial to gain a basic understanding of the conflicting values veterans encounter during their transition from civilian life to the stressors of war and back again [38]. This can be done through joining the Wounded Warrior Project (WWP) (woundedwarriorproject.org) and reading relevant articles and personal biographies of veterans and families.

\section{Advocacy}

Advocacy can help address veterans' needs and reduce the stigma associated with mental health care [11]. Three ways to do so are to stay informed with current events and legislation, attend military deployment and post-deployment events, and donate funding for veterans' programs.

Veteran support organizations like the WWP and Soldiers Angels (soldiersangels.org) use their websites for veterans and their families to stay informed about research, treatment options, donation opportunities (such as money, food, or time), and legislation. New legislation will increase the resources available to veterans and families, and clinicians connected with these organizations can stay on top of new developments.

Showing support at a local level (such as through participating in marathons, fundraisers, or concerts) is supportive and apolitical. For clinicians who wish to advocate for this population, being present at such events is an essential step in helping this population feel acknowledged, appreciated, and accepted. These events are listed at woundedwarriorproject.org.

\section{Conclusion}

Soldiers often endure complicated, difficult, and life-altering physical and mental health problems during and after their service [8]. Clinicians must address these unique needs using evidence-based practices, while remaining sensitive to their clients' emotional and cultural needs. Clinicians' ability to administer ethical, high-quality care will afford veterans and their families the best possible opportunities to heal and become whole. The behavioral health field is faced with a dynamic opportunity to address the veteran population's needs head-on through advocacy efforts and raise awareness to gain support. Doing so is a major step toward reducing the stigma associated with mental health care and that will help veterans enjoy the life for which they fought, and the life they deserve.

\section{References}

1. Taft CT, Kaloupek DG, Schumm JA, Marshall AD, Panuzio J, et al. (2007) Posttraumatic stress disorder symptoms, physiological reactivity, alcohol problems, and aggression among military veterans. J Abnorm Psychol 116: 498-507.

2. Seal K, Metzler T, Gima K, Bertenthal D, Maguen S, et al. (2009) Trends and risk factors for mental health diagnoses among Iraq and Afghanistan veterans using department of veterans affairs health care, 2002-2008. Am J Public Health 99: 1651-1658.

3. Stirman S (2008) The applicability of randomized controlled trials of psychosocial treatments for PTSD to a veteran population. J Psychiatr Pract 14: 199-208.

4. Hendin H, Maltsberger JT, Szanto K (2008) The psychosocial context of trauma in treating PTSD patients. Am J Psychiatry 165: 28-32.

5. Wessa MCA, Flor H (2007) Failure of extinction of fear responses in posttraumatic stress disorder: Evidence from second-order conditioning. Am J Psychiatry 164: 1684-1692.

6. Karlin BE, Ruzek JI, Chard KM, Eftekhari A, Monson CM, et al. (2010) Dissemination of evidence-based psychological treatments for posttraumatic stress disorder in the Veterans Health Administration. J Trauma Stress 23: 663-673.

7. Nash WP, Silva C, Litz B (2009) The historic origins of military and veteran mental health stigma and the stress injury model as a means to reduce it. Psychiatr Ann 39: 789-794.

8. Ouimette PC, Vogt D, Wade M, Tirone V, Greenbaum MA, et al. (2011) Perceived barriers to care among veterans health administration patients with posttraumatic stress disorder. Psychiatr Serv 8: 212-223.

9. Talbott J (2006) Combat duty in Iraq and Afghanistan, mental health problems, and barriers to care. Yearbook of Psychiatry and Applied Mental Health 142-144.

10. Luxton D, Skopp N, Maguen S (2010) Gender differences in depression and PTSD symptoms following combat exposure. Depress Anxiety 27: 1027-1033.

11. Vogt D (2011) Mental health-related beliefs as a barrier to service use for military personnel and veterans: A review. Psychiatr Serv 62: 135-142.

12. Magruder KM, Yeager DE (2009) The prevalence of PTSD across war eras and the effect of deployment on PTSD: A systematic review and metaanalysis. Psychiatr Ann 39: 778-788.

13. Schnurr PP, Lunney CA, Sengupta A (2004) Risk factors for the development versus maintenance of posttraumatic stress disorder. J Trauma Stress 17: 85-95.

14. Maguen S, Lucenko BA, Reger MA, Gahm GA, Litz BT, et al. (2010) The impact of reported direct and indirect killing on mental health symptoms in Iraq war veterans. J Trauma Stress 23: 86-90.

15. Wenzel A, Brown GK, Karlin BE (2011) Cognitive behavioral therapy for depression in veterans and military service members: Therapist Manual. U.S. Department of Veterans Affairs, Washington, DC. 
Citation: McTighe AJ (2017) Evidence-Based Practices, Ethical Considerations, and Advocacy Efforts to Reduce Mental Health Stigma in Veterans with Post-Traumatic Stress Disorder. J Trauma Treat 6: 398. doi:10.4172/2167-1222.1000398

Page 5 of 5

16. Jakupcak M, Vannoy S, Imel Z, Cook JW, Fontana A, et al. (2010) Does PTSD moderate the relationship between social support and suicide risk in Iraq and Afghanistan War Veterans seeking mental health treatment? Depress Anxiety 27:1001-1005.

17. Zuiden MV, Geuze E, Willemen HL, Vermetten E, Maas M, et al. (2011) Pre-Existing high glucocorticoid receptor number predicting development of posttraumatic stress symptoms after military deployment. Am J Psychiatry 168: 89-96.

18. Possemato K, Wade M, Andersen J, Ouimette P (2010) The impact of PTSD, depression, and substance use disorders on disease burden and health care utilization among OEF/OIF veterans. Psychol Trauma 2: 218-223.

19. DMDC (2011) Workplace and gender relations survey of active duty members. Arlington.

20. Dutra L, Grubbs K, Greene C, Trego LL, McCartin TL, et al. (2011) Women at war: Implications for mental health. J Trauma Dissociation 12: 25-37.

21. Bakken T (2002) The effects of hate crime legislation: unproven benefits and unintended consequences. International Journal of Discrimination and the Law 5: 231-246.

22. Ghafoori B, Hierholzer RW (2010) Personality patterns among Black, White, and Hispanic combat veterans. Psychol Trauma 2: 12-18.

23. Smith-Osborne A (2009) Mental health risk and social ecological variables associated with educational attainment for gulf war veterans: Implications for veterans returning to civilian life. Am J Community Psychol 44: 327-337.

24. Highfill-Mcroy RM, Larson GE, Booth-Kewley S, Garland CF (2010) Psychiatric diagnoses and punishment for misconduct: the effects of PTSD in combat-deployed Marines. BMC Psychiatry 10: 88.

25. Resick PA, Monson CM, Rizvi SL (2008) Posttraumatic stress disorder. In D. H. Barlow, D. H. Barlow (Eds.), Clinical handbook of psychological disorders: A step-by-step treatment manual (4th edn) Guilford Press, New York. pp. 65-122.

26. Frueh BC, Monnier J, Grubaugh AL, Elhai JD, Yim E, et al. (2007) Therapist adherence and competence with manualized cognitivebehavioral therapy for PTSD delivered via videoconferencing technology. Behav Modif 31: 856-866.
27. Chard KM, Schumm JA, Owens GP, Cottingham SM (2010) A comparison of OEF and OIF veterans and Vietnam veterans receiving cognitive processing therapy. J Trauma Stress 23: 25-32.

28. Tuerk PW, Grubaugh AL, Hamner MB, Foa EB (2009) Diagnosis and treatment of PTSD-Related compulsive checking behaviors in veterans of the Iraq war: The influence of military context on the expression of PTSD symptoms. Am J Psychiatry 166: 762-767.

29. Foa EB, Cahill SP, Boscarino JA, Hobfoll SE, Lahad M, et al. (2005) Social, psychological, and psychiatric interventions following terrorist attacks: Recommendations for practice and research. Neuropsychopharmacology 30: 1806-1817.

30. Strachan M, Gros DF, Ruggiero KJ, Lejuez CW, Acierno R (2012) An integrated approach to delivering exposure-based treatment for symptoms of PTSD and depression in OIF/OEF veterans: Preliminary findings. Behav Ther 43: 560-569.

31. Rothbaum BO, Astin MC, Marsteller F (2005) Prolonged exposure versus eye movement desensitization and reprocessing (EMDR) for PTSD rape victims. J Trauma Stress 18: 607-616.

32. Holmes EA, Arntz A, Smucker MR (2007) Imagery rescripting in cognitive behavior therapy: images, treatment techniques and outcomes. J Behav Ther Exp Psychiatry 38: 297-305.

33. Long ME, Quevillon R (2009) Imagery rescripting in the treatment of posttraumatic stress disorder. J Cogn Ther 23: 67-76.

34. Juretic M, Hill R, Luptak M, Rupper R, Bair B, et al. (2010) Reaching out to older veterans in need: The elko clinic demonstration project. J Rural Health 26: 325-332.

35. Knapp S, VandeCreek LD (2012) Practical ethics for psychologists: a positive approach.

36. Orchowski LM, Spickard BA, Mcnamara JR (2006) Cinema and the valuing of psychotherapy: Implications for clinical practice. Professional Psychology: Research and Practice 37: 506-514.

37. Mckay D (2000) Stigmatizing pharmaceutical advertisements. Br J Psychiatry 177: 467-468.

38. Beshai JA, Tushup RJ (2013) Sanctity of human life in war: Ethics and post-traumatic stress disorder. Applied Ethics in Mental Health Care $125-134$. 